\title{
MEJORA DE PROCESOS PARA MITIGAR \\ EL IMPACTO AMBIENTAL \\ EN UNA LAVANDERÍA INDUSTRIAL
}

WALTER MONTOYA*

Pontificia Universidad Católica del Perú, Perú

Recibido: 17 de febrero del 2020 / Aprobado: 24 de abril del 2020

doi: 10.26439/ing.ind2020.n038.4816

RESUMEN: Hoy en día no basta con disminuir los costos o incrementar la productividad, también es necesario enfocar las mejoras de los procesos en mitigar el impacto ambiental generado. Por ello, se analizó la situación actual de una lavandería industrial llamada WALU, empleando como metodología de diagnóstico la matriz de identificación de riesgos ambientales para proponer mejoras que mitiguen y eliminen el impacto ambiental, consiguiendo cambiar el modelo de negocio a uno sostenible.

Palabras clave: lavandería industrial / proceso de mejora continua / análisis del impacto ambiental

\section{PROCESS IMPROVEMENT FOR ENVIRONMENTAL IMPACT MITIGATION IN AN INDUSTRIAL LAUNDRY}

ABSTRACT: Nowadays it is not enough to reduce costs or increase productivity; it is also necessary to focus process improvements on mitigating the environmental impact. For this reason, the current situation of the WALU industrial laundry was analyzed using the environmental risk identification matrix as diagnostic methodology to propose improvements that mitigate and eliminate the environmental impact, changing the business model to a sustainable one.

Keywords: industrial laundry / continuous improvement process / environmental impact analysis

*montoya.wm@pucp.pe 


\section{INTRODUCCIÓN}

El cambio climático es uno de los grandes problemas en la actualidad con efectos en los índices macroeconómicos, por ejemplo, en la disminución del PBI o en el incremento de la pobreza; afectando, principalmente, a países que poseen una gran variedad de ecosistemas, como es el caso del Perú, donde las pérdidas proyectadas por el cambio climático, para el año 2025, podrían ser de unos 9906 millones de dólares (Cueva, 2014), por lo que debe ser tenido en cuenta.

Este tipo de impacto de carácter ambiental ha traído como consecuencia la necesidad de estar alineados con nuevas regulaciones estatales que consideran el cuidado del medio ambiente y exigen que se busquen mejoras que ayuden a mitigar el impacto ambiental que generan las empresas para que se pueda vivir en un ambiente saludable y equilibrado (Ley n. ${ }^{\circ} 28611$, Ley General del Ambiente, 2005).

Por ello, el presente artículo tiene como objetivo mejorar los procesos de una lavandería industrial, WALU, la cual ofrece diferentes servicios de lavado para prendas de vestir, tanto de tela denim y dril, enfocándose en aquellas mejoras que mitiguen el impacto ambiental.

\section{METODOLOGÍA}

La metodología a emplear es la matriz de identificación de riesgo ambiental (matriz IRA), con la cual se realizan diagnósticos sobre la situación actual de los procesos de las empresas, teniendo en cuenta todas las causas y efectos generados. La matriz IRA está basada en la norma NTP-ISO 14001:2015 (2015) y en la metodología de evaluación de aspectos ambientales de Chauvet, Bello, Barnes y Albarracín (2014).

En comparación con otras metodologías que evalúan el riesgo ambiental, la matriz IRA permite determinar el índice de riesgo ambiental considerando 4 factores: a) el alcance del efecto generado (el radio de acción), b) la frecuencia de la presencia del aspecto ambiental en el proceso, c) el control existente en el proceso; y d) la severidad o gravedad del efecto generado, es decir, la magnitud de las consecuencias.

La matriz IRA ordena los aspectos ambientales y los impactos ambientales por proceso. Entiéndase por aspecto ambiental toda causa o fuente que genere un efecto al que llamamos impacto ambiental. Por ejemplo, cuando se lava la ropa, se emplea agua y detergente; y como elemento de salida se observa efluentes de agua con detergente. Entonces, la causa o el aspecto ambiental sería la generación de efluentes de agua con detergente y esto trae, como consecuencia, el impacto ambiental de contaminación del agua. 


\section{DESCRIPCIÓN DE LA EMPRESA}

WALU es el nombre ficticio de una empresa peruana del sector industrial-textil, especializada en lavados, teñidos y tratamientos de prendas de vestir de tela denim y dril. WALU inició sus actividades hace nueve años en el distrito de San Martín de Porres, en la ciudad de Lima, y actualmente es reconocida por la calidad en los servicios que ofrece no solo en Lima sino a lo largo del Perú (ciudades como Chiclayo, Trujillo y Piura). Tiene como cliente final a empresas del sector textil que poseen volúmenes de confección con lotes entre 500 y 1000 prendas de vestir. Actualmente, WALU maneja una producción mensual de 30000 prendas de vestir tratadas entre los diferentes tipos de lavados que realiza.

\section{RESULTADOS}

En esta sección se presenta la evaluación de aspectos e impactos ambientales empleando la matriz IRA y las propuestas de mejora.

\subsection{Procesos de la lavandería industrial}

Los procesos que siguen las prendas de vestir comienzan con la recepción de las mismas. Estas son preparadas de acuerdo con las necesidades del cliente. Por ejemplo, en un lote de 300 prendas, si el cliente requiere 3 colores, se preparan 3 lotes de 100. En caso de que requieran algún arreglo manual, como cortes, estos se realizan, también, en el preparado.

Si el lavado lo requiere, pasan al proceso de focalizado, donde se aplican químicos para poder generar un efecto de blanqueado y desgaste en las prendas de vestir. Luego, se envían al proceso de lavado mediante la aplicación de diferentes químicos, agua y vapor, así, mediante los diferentes enjuagues se consigue el efecto deseado, como el teñido. Para extraer el agua de las prendas de vestir, pasan por el proceso de centrifugado, y para secarlas son transportadas al proceso de secado.

Finalmente, son planchadas y empaquetadas. El último proceso es una inspección visual, en caso de que una prenda de vestir tenga un defecto, un color distinto o un corte, es separada del lote.

A modo de resumen en la figura 1 se muestra el diagrama de flujo de los procesos anteriormente descritos. 


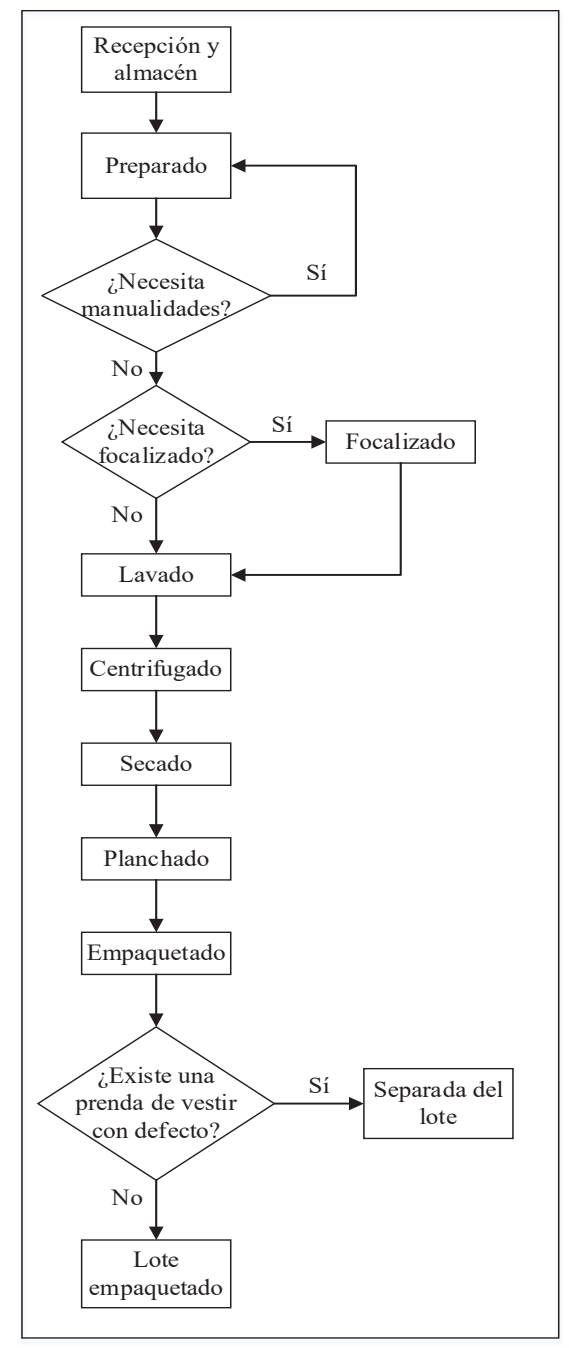

Figura 1. Diagrama de flujo de los procesos

Elaboración propia

\subsection{Identificación y evaluación de los aspectos e impactos ambientales}

Para poder identificar los aspectos e impactos ambientales, se emplea la matriz IRA. En la tabla 1 se muestran los elementos de entrada y de salida de cada actividad; también se identifican los aspectos e impactos ambientales de cada uno de estos procesos. La tabla 2 contiene la evaluación de cada uno de estos impactos, demostrando así cuales son significativos, aquellos con mayores efectos de relevancia. Cabe resaltar que las actividades están ordenadas de acuerdo con el flujograma de la figura 1. 


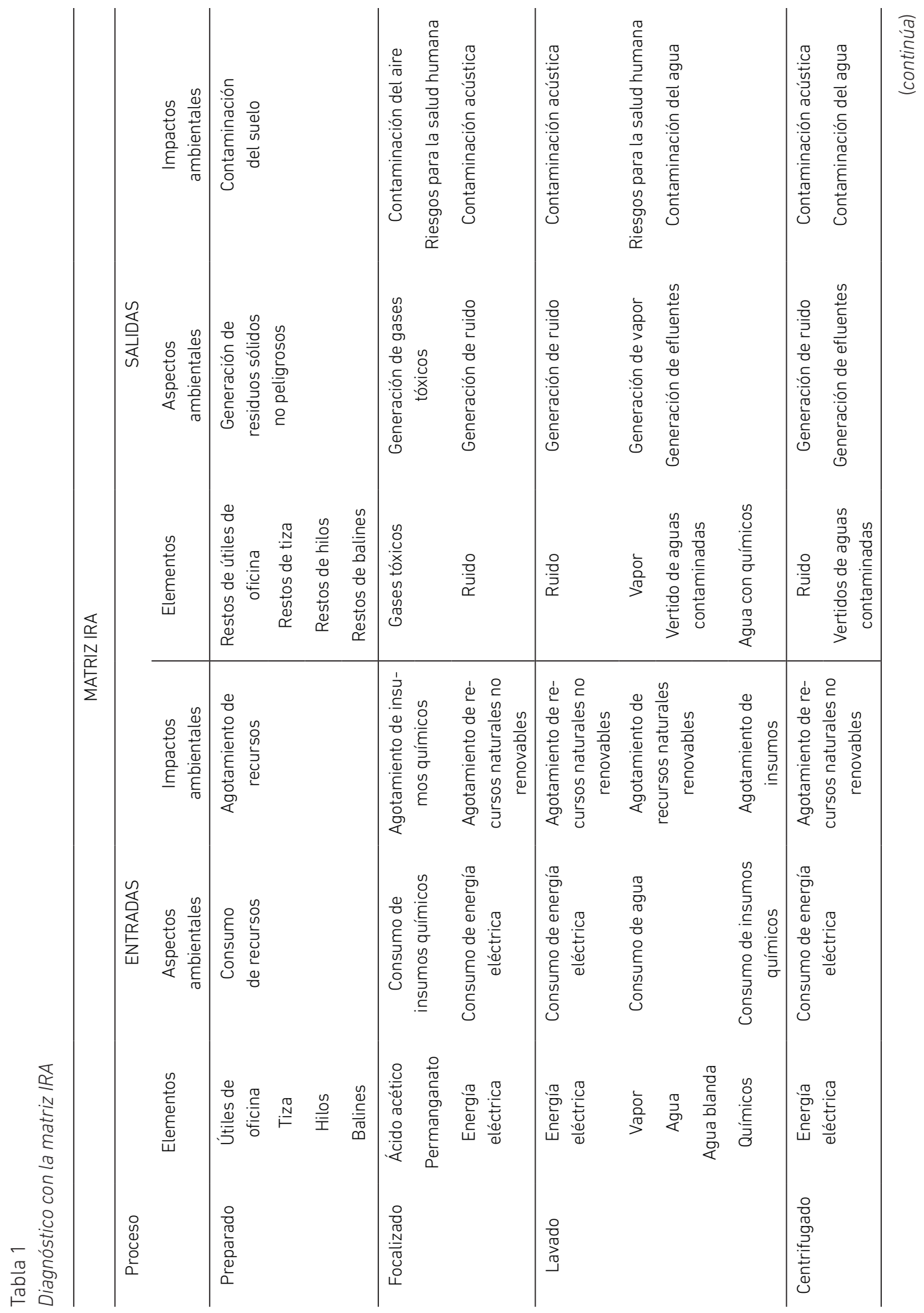


Walter Montoya

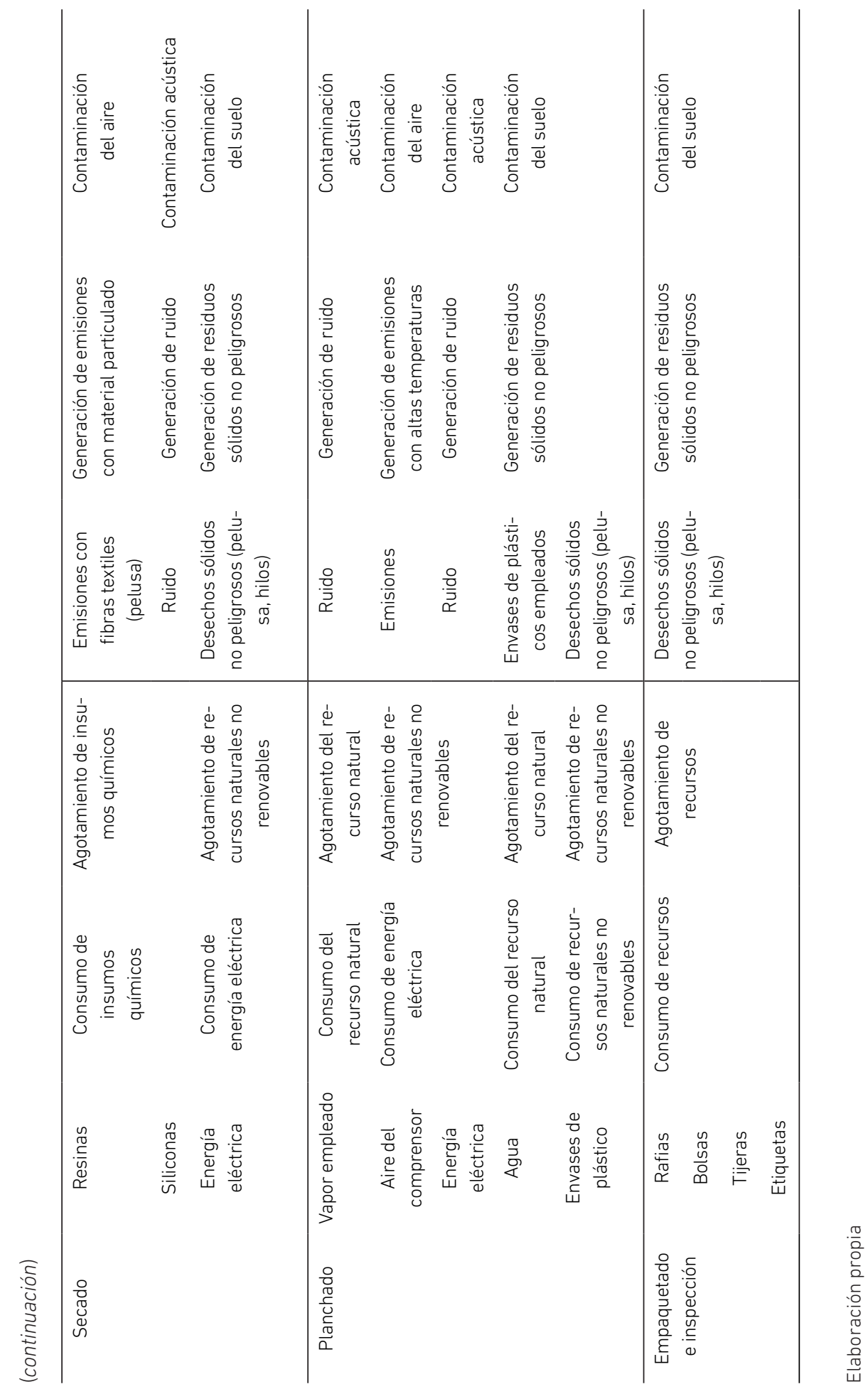


Mejora de procesos para mitigar el impacto ambiental en una lavandería industrial

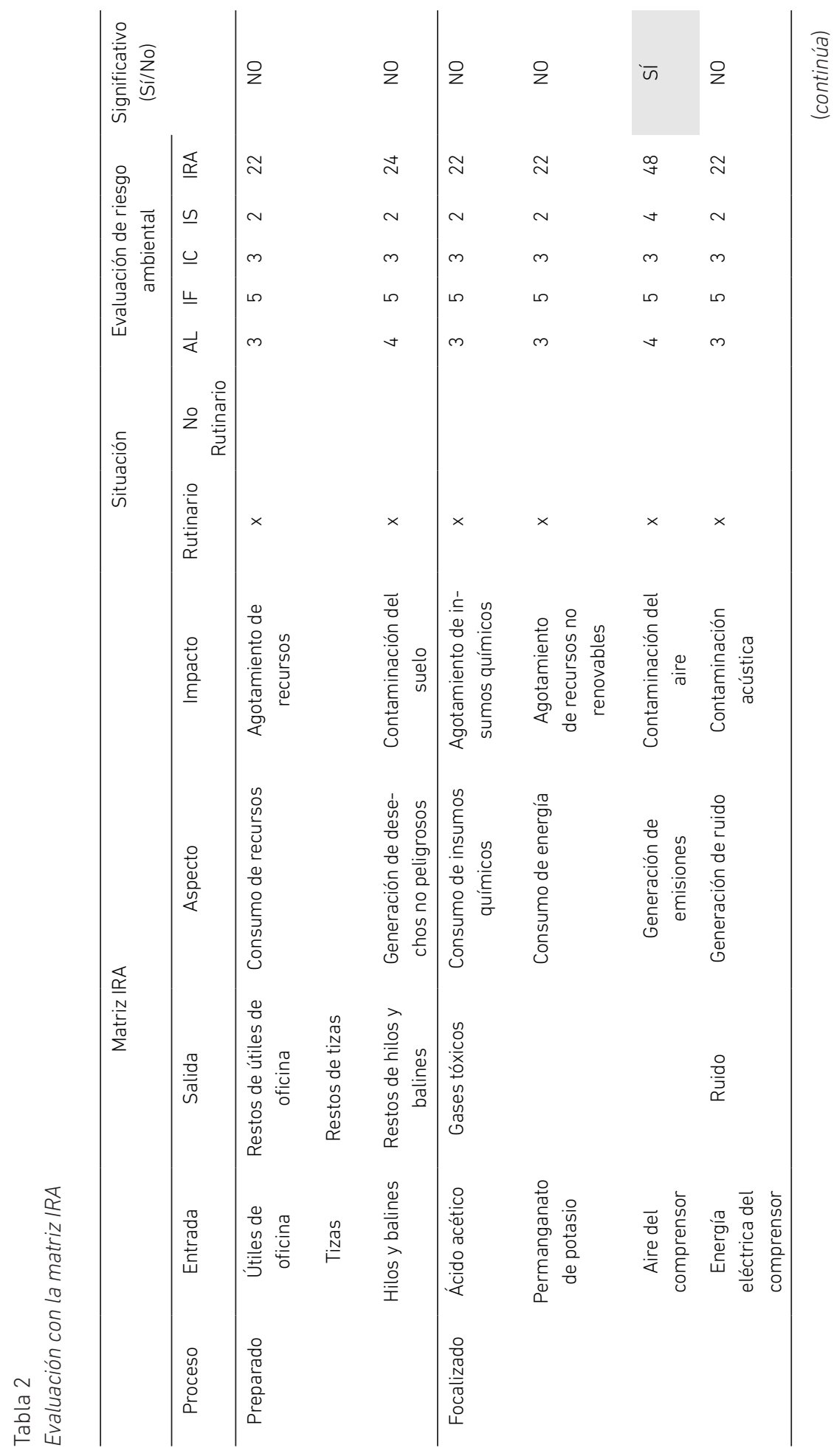


Walter Montoya

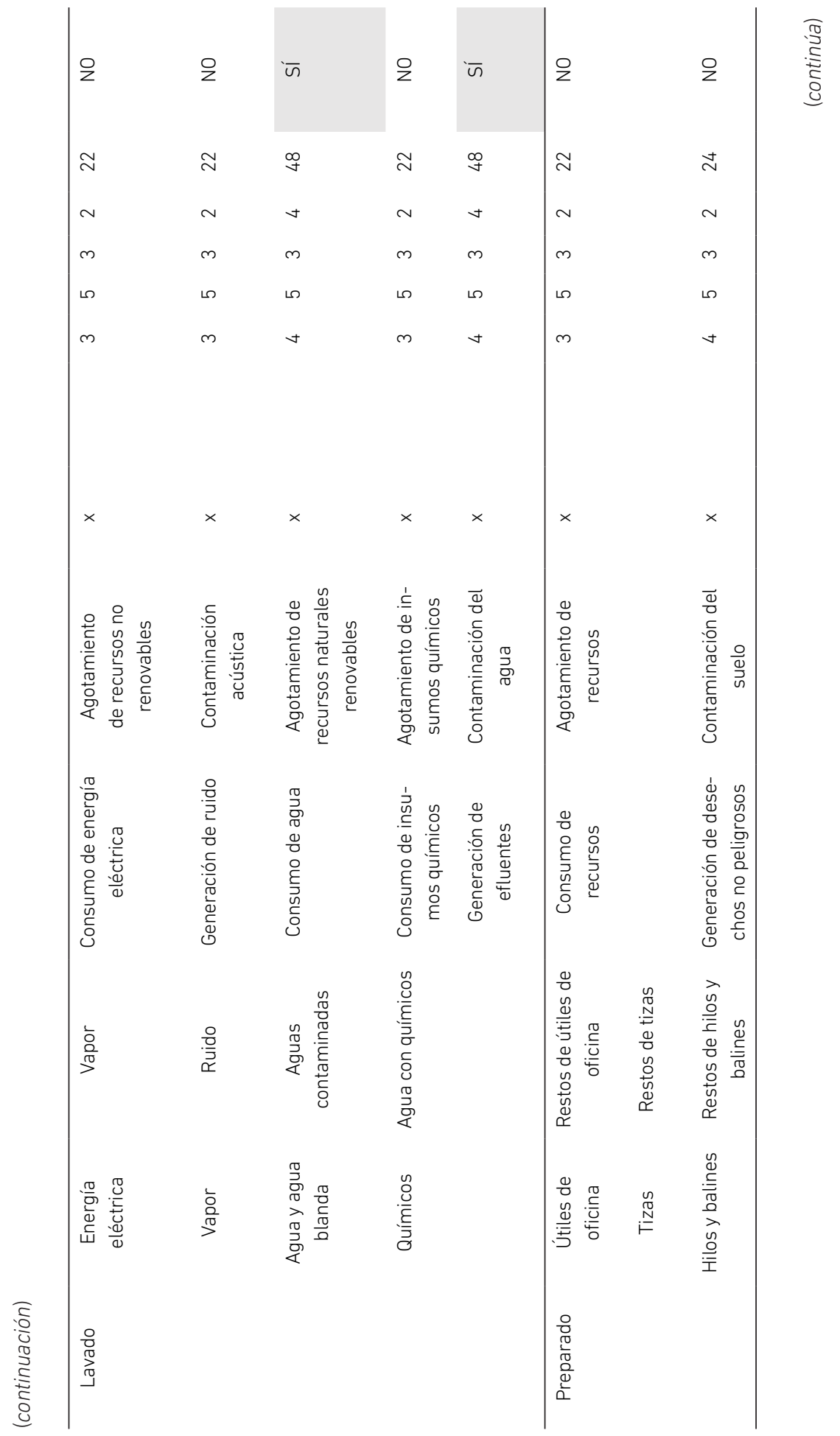


Mejora de procesos para mitigar el impacto ambiental en una lavandería industrial

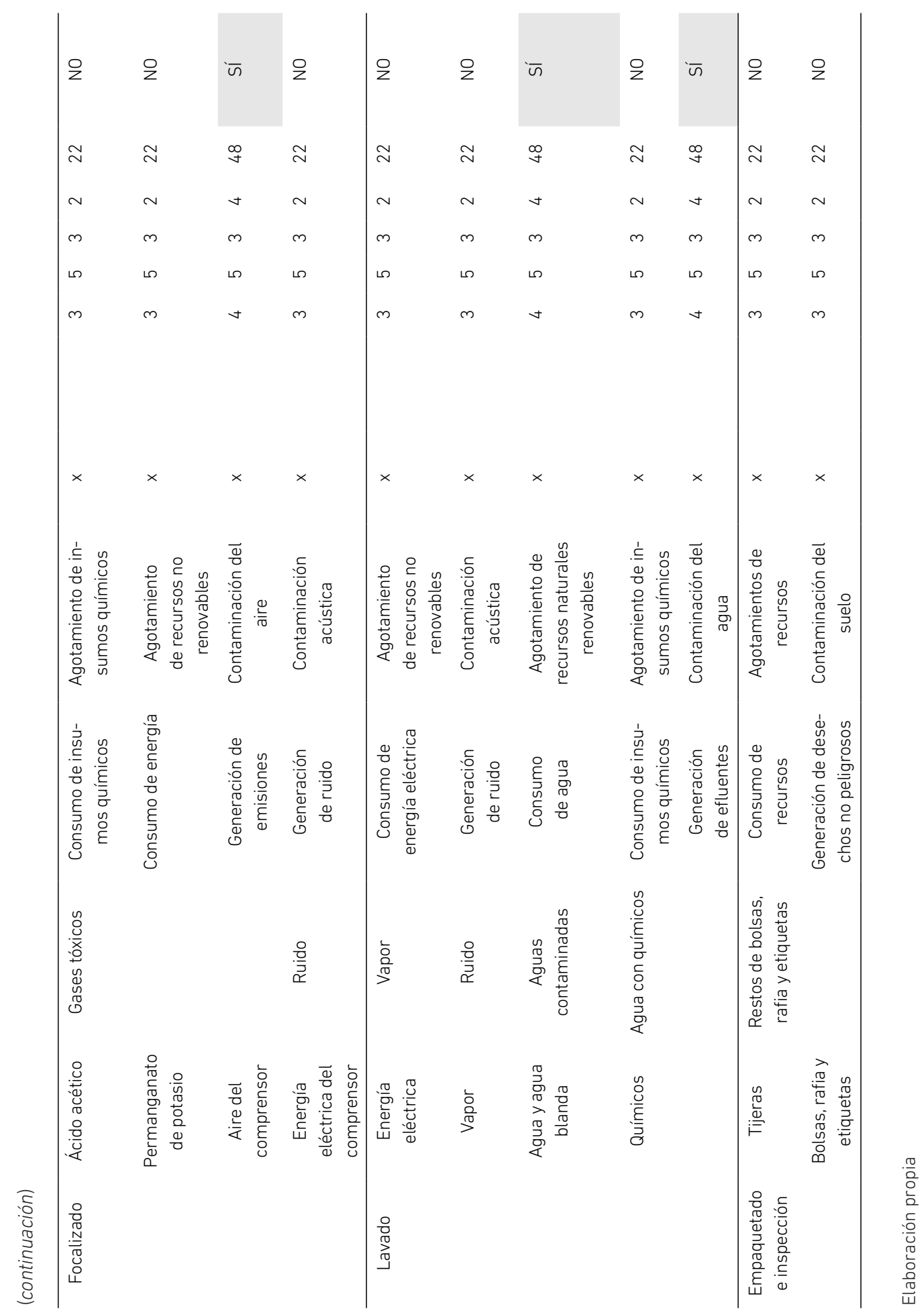


Se puede observar que la empresa genera diferentes impactos ambientales a lo largo de los procesos que realiza. Además, son significativos, pues poseen un valor del indicador IRA mayor a 33.

Los impactos ambientales más relevantes son: la generación de los gases tóxicos generados en el proceso de focalizado, la generación de efluentes con químicos empleados en los procesos de lavado, la emisión de aire con material particulado del proceso de secado y el consumo de agua en los reprocesos en la operación de lavado.

Estos impactos se deben, principalmente, a una falta de políticas; por ejemplo, existe una falta de control de los efluentes generados, que trae como consecuencia la contaminación del agua y problemas con entidades estatales como Sedapal.

A continuación, se agrupa y se detalla el impacto ambiental según el agente que contamina para un mejor entendimiento del mismo:

\section{- Generación de emisiones}

Respecto al proceso de focalizado, los problemas a resolver son los gases generados. Estos gases son expulsados libremente en la fábrica. Por otro lado, en el proceso de secado, existe una falta de filtros adecuados para retener la pelusa que se expulsa al medioambiente por las salidas de aire de las máquinas secadoras, ya que actualmente solo una parte de este aire con pelusa es direccionada al desagüe y otra termina en la fábrica misma; por ello, se necesita una renovación de las partes (filtros y canales de salidas), pues existen filtraciones.

\section{- Generación de efluentes}

Se requiere un control de los efluentes generados, ya que el volumen de agua que se vierte al desagüe es significativo y diario. Estos efluentes son una combinación de agua con detergentes, químicos de los lavados y, en ciertos casos, pelusa. Es decir, no se le realiza tratamiento alguno y, además, de contaminar el agua, estos deterioran la infraestructura del alcantarillado.

Además, existen otros impactos de menor significancia, pero constantes en diferentes procesos. Uno de estos es la generación del ruido que cuenta con un indicador IRA de 22, ya que el impacto es solo alrededor de la fábrica. Esto se debe a que las máquinas empleadas cuentan con cierta antigüedad (entre 8-10 años) y no son completamente automatizadas. Los cambios son manuales y los procesos que realizan generan ruido en la fábrica. Para solucionar este problema y evitar algún impacto en los operarios, se necesita emplear equipos de protección personal (EPP) como audífonos. 


\subsection{Propuestas de mejora}

En esta sección se describen las propuestas de mejora para los aspectos ambientales significativos identificados, anteriormente, con las respectivas metas y ecoindicadores, tanto para la situación actual como para la mejorada. Asimismo, es necesario ejecutar solo aquel conjunto de mejoras que genere el mayor beneficio; por ello, las mejoras propuestas para la empresa cumplen con los siguientes criterios:

- Produce un menor impacto ambiental en comparación con la situación actual

- No afecta al costo de producción actual

- No implica mayor efecto en otras áreas (impacto en la velocidad de producción)

- Genera un menor costo de mantenimiento

- No requiere personal extra

- Necesita una menor inversión inicial

\subsubsection{Mejora para la operación de focalizado}

El focalizado es una actividad ampliamente usada en la industria textil, ya que el efecto generado es aún parte de las tendencias de moda. Tiene como aspecto ambiental las emisiones con componentes tóxicos $\left(\mathrm{CH}_{3} \mathrm{COOH}+\mathrm{KOH}+\mathrm{MNO}_{2}\right)$ que traen como impacto ambiental la contaminación del aire, afectando a la salud humana. Por ello, se establece como meta evitar la contaminación del aire por parte del proceso de focalizado.

La solución que emplean las lavanderías industriales es el uso de maquinarias con tecnología láser, que generan el mismo efecto, pero haciendo uso solo de energía eléctrica como elemento de entrada para el funcionamiento de la máquina; como elemento de salida, luego de haber realizado el proceso, se genera vapor de la tela que ha sido desgastada. No se aplica químico alguno y los procedimientos tienen que ser prediseñados para evitar cualquier accidente como las quemaduras. El problema con esta tecnología es la inversión inicial de la maquinaria y de la infraestructura que se necesita. Sin embargo, aplicándola, se eliminaría por completo el impacto ambiental generado por el proceso de focalizado y se evitaría la compra de insumos tóxicos.

Para poder evaluar si el impacto ambiental del proceso es mitigado, se proponen los siguientes ecoindicadores:

- Kilogramo de permanganato de potasio al mes

- Kilogramo de ácido acético al mes

Esto con el fin de poder analizar la cantidad de químicos empleados de acuerdo con el nivel de producción. Además, con la aplicación de esta mejora se pronostica el escenario mostrado en la tabla 3 . 
Sin embargo, mientras aún se conserve el actual método de trabajo para la operación de focalizado, se recomienda adquirir insumos con mejor calidad, de manera que se emplee solamente lo necesario y así evitar la generación de gases tóxicos innecesarios.

Tabla 3

Situación actual y mejorada del proceso de focalizado

\begin{tabular}{ll}
\hline \multicolumn{1}{c}{ Propuesta } & \multicolumn{1}{c}{ Implementación de una máquina láser } \\
\hline Situación actual de los ecoindicadores & 1. Kilogramo de permanganato de potasio al mes: 80 \\
& 2. Kilogramo ácido acético al mes: 70 \\
Situación mejorada de los & 1. Kilogramo de permanganato de potasio al mes: 0 \\
ecoindicadores & 2. Kilogramo ácido acético al mes: 0 \\
\hline
\end{tabular}

Elaboración propia

\subsubsection{Mejoras para la operación de secado}

Actualmente, los equipos del proceso de secado no son los más eficientes, ya que generan un ambiente desordenado y lleno de material particulado, afectando directamente a la salud de los colaboradores; por ello, la meta es poder liberar aire purificado. El aspecto ambiental es la generación de emisiones con material particulado (pelusa), y el impacto ambiental es la contaminación del aire.

Para poder mejorar la situación, se presentan las siguientes mejoras.

Primero, las máquinas secadoras poseen filtros y radiadores. Actualmente, no existen procedimientos predefinidos para su mantenimiento. Por lo que se propone, a modo de política, una frecuencia semanal de limpieza para los filtros de aire y una mensual para los radiadores, de manera que se asegure el óptimo estado de los mismos. Vale resaltar que el operario debe emplear los EPP correspondientes (mascarilla y lentes) para evitar cualquier accidente.

Luego, se propone reemplazar los canales de salida de aire, que actualmente son ductos con material de plástico, por ductos de lata cromada. De manera que se evita tener alguna fuga de aire para así direccionar completamente el flujo hacia el exterior y evitar cualquier filtración o incidente.

Implementando las dos medidas anteriores, se puede afirmar que el impacto de las máquinas secadoras es el mínimo, ya que solo emitiría aire y la pelusa sería retenida. Con la aplicación de esta mejora se pronostica el escenario mostrado en la tabla 4.

Por ello, se propone, a modo de política, verificar la limpieza del aire mediante una prueba de concentración del material particulado $\left(\mu \mathrm{g} / \mathrm{m}^{3}\right)$, así poder evaluar si el impacto ambiental del proceso es menor. 
Tabla 4

Situación actual y mejorada del proceso de secado

\begin{tabular}{ll}
\hline \multicolumn{1}{c}{ Propuesta } & Implementación de ductos de aire de metal y limpieza de filtros \\
\hline Situación actual de los ecoindicadores & 1. Concentración del material particulado $(\mu \mathrm{g} / \mathrm{m} 3): 0,5$ \\
$\begin{array}{l}\text { Situación mejorada de los } \\
\text { ecoindicadores }\end{array}$ & 1. Concentración del material particulado $(\mu \mathrm{g} / \mathrm{m} 3): 0$ \\
\hline
\end{tabular}

Elaboración propia

\subsubsection{Mejora para la operación de lavado}

El proceso de lavado es la operación que emplea la mayor cantidad de recursos; especialmente, el agua, ya que esta es la materia prima para los diferentes enjuagues. Tiene como aspecto ambiental el consumo de agua (entre 2500-3200 litros semanales) y como impacto ambiental el agotamiento del recurso hídrico. Por ello, se tiene que establecer como meta ser eficientes en el empleo del agua.

Actualmente no se visualiza deterioro en tuberías ni en llaves que proveen de agua blanda a las máquinas lavadoras; por ello, se necesita proponer una política de mantenimiento mensual para poder mantener el estado óptimo de la infraestructura, con el fin de evitar cualquier fuga de agua. Esta inspección se puede realizar de manera visual y es una propuesta de mejora a modo de mantenimiento preventivo.

Por otro lado, la existencia de reprocesos no solo genera un empleo adicional de químicos y de energía eléctrica, sino también de agua blanda, por lo que se propone un cuidado especial en los procesos de lavado y centrifugado. La mayoría de dichos reprocesos suceden cuando las prendas se "betan"; es decir, se destiñen entre sí porque no fueron conducidas al proceso de secado, luego de haber sido centrifugadas. Por lo que debe designarse un operario para que asegure de que ningún lote se llegue a betar, al tiempo deben capacitarse los demás operarios sobre las consecuencias de los reprocesos y así evitarlos en su totalidad.

Para evaluar si se ha mitigado el impacto ambiental, se proponen los siguientes ecoindicadores.

- Litros de agua blanda/kilogramos de prendas de vestir

- Cantidad de reprocesos al mes

Con el fin de analizar la cantidad de agua blanda empleada por la cantidad de prendas de vestir a un determinado nivel de producción y el número de reprocesos. Con la aplicación de esta mejora se pronostica el escenario mostrado en la tabla 5. 
Tabla 5

Situación actual y mejorada del proceso de lavado

\begin{tabular}{ll}
\hline \multicolumn{1}{c}{ Propuesta } & Designar un personal especializado y capacitar a los operarios \\
\hline Situación actual de los ecoindicadores & 1. Litros de agua/kilogramos de prendas de vestir: 2 \\
& 2. Cantidad de reprocesos al mes: 5 \\
Situación mejorada de los & 1. Litros de agua/kilogramos de prendas de vestir: 1,5 \\
ecoindicadores & 2. Cantidad de reprocesos al mes: 0 \\
\hline
\end{tabular}

Elaboración propia

\subsubsection{Mejora para la operación de lavado y centrifugado}

Tanto el proceso de lavado como el de centrifugado, tienen como principal elemento de salida los efluentes con los distintos químicos empleados para el teñido de las prendas de vestir. Estos efluentes tienen que ser tratados antes de ser vertidos al desagüe. Se tiene como aspecto ambiental la generación de efluentes con alto nivel de $\mathrm{pH}$, de demanda bioquímica de oxígeno (DBO) y de demanda química de oxígeno (DQO); y como impacto ambiental la contaminación del agua. Por ello, se tiene como meta reducir la presencia de químicos en los efluentes y alinearse a los valores máximos admisibles (VMA) para descargas de aguas residuales no domésticas.

En la actualidad no se monitorea ningún indicador de los efluentes. Por ello se propone una política para la toma de los indicadores con frecuencia semanal. Estos indicadores se deben analizar según los estándares propuestos para los VMA para efluentes

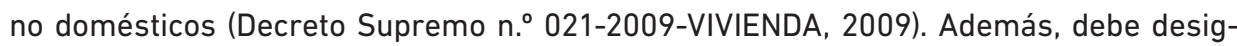
narse a un operario especial y capacitarlo correctamente; también, considerar que el operario debe emplear los EPP correspondientes, como guantes y lentes, y poseer las herramientas debidamente calibradas para la toma de muestras y la evaluación de las mismas.

Se tienen que medir los principales indicadores (Monllor, Sanz, Vicente y Bonet, 2013):

- $\quad$ El DQO, que debe tener un valor máximo de 1000 mg/L (MVCS, 2009)

- $\quad$ El DBO, que debe tener un valor máximo de 500 mg/L (MVCS, 2009)

- El nivel de pH, que debe estar entre un rango de 6-9 (MVCS, 2009)

El indicador de DBO, actualmente, está estable, con un valor de $399,5 \mathrm{mg} / \mathrm{L}$ (Monllor et al., 2013); sin embargo, es necesario realizarle un control por ser uno de los principales indicadores de los efluentes textiles (Monllor et al., 2013). 
Luego, para poder corregir los otros indicadores, se propone cambiar la infraestructura actual, como se muestra en la figura 2, a un sistema de tratamientos de aguas residuales, para eliminar los químicos y contaminantes presentes en los efluentes, como se muestra en la figura 3. A continuación, se explica el sistema propuesto.

Primero, se necesita una etapa de regulación de caudal. Por lo tanto, se propone colocar un pozo de unión de caudales. Luego, como un pretratamiento, se tiene que evitar la presencia de sólidos en los efluentes, en todo momento, ya que ellos pueden obstruir el alcantarillado generando inundaciones y afectando la infraestructura de la planta. La mayoría de estos sólidos son hilos y pelusas de las prendas de vestir. Por ello, en un segundo pozo, se sugiere la implementación de filtros que retengan todos los hilos y las pelusas existentes, para poder retener todo material sólido. Además, como política, realizar un chequeo diario de los filtros, para la limpieza y la verificación del estado óptimo de estos.

Luego, para el control de químicos, en especial de metales pesados, por medio de un filtro de carbón activo, con una capacidad de $20 \mathrm{~m} / 3 / \mathrm{h}$, se pueden generar efluentes con menor presencia de estos (DeJohn y Hutchins, 1976), de manera que estén dentro del rango permitido de los VMA para efluentes no domésticos.

Para poder generar un ahorro, se propone instalar un filtro de osmosis con una capacidad de $6 \mathrm{~m}^{3} / \mathrm{h}$, junto con un tanque de $25 \mathrm{~m}^{3}$ para la regularización del caudal. Así se reutilizaría el agua, y el ahorro representaría el $30 \%$ de los efluentes generados. Luego, con el uso de este último filtro, el nivel de DBO y de DQO sería menor (Brandon, Johnson, Minturn y Porter, 1973).

Asimismo, se instalaría un tanque de $5 \mathrm{~m}^{3}$ para almacenar los residuos acuosos generados por los filtros de carbón activado y osmosis. Este estaría almacenado temporalmente hasta que una empresa operadora de residuos sólidos lo recoja. Entonces, para evaluar el estado del impacto ambiental se proponen los siguientes ecoindicadores.

- $\quad$ El DBO en $\mathrm{mg} / \mathrm{L}$

- $\quad E l D Q O$ en $\mathrm{mg} / \mathrm{L}$

- $\quad$ El nivel de $\mathrm{pH}$

Con la aplicación de esta mejora se pronostica el escenario mostrado en la tabla 6. 
Walter Montoya

Tabla 6

Situación actual y mejorada del proceso de lavado y centrifugado

\begin{tabular}{ll}
\hline \multicolumn{1}{c}{ Propuesta } & \multicolumn{1}{c}{$\begin{array}{c}\text { Implementación de un sistema de } \\
\text { tratamiento de aguas residuales }\end{array}$} \\
\hline $\begin{array}{l}\text { Situación actual de los } \\
\text { ecoindicadores }\end{array}$ & $\begin{array}{l}\text { 1. DBO: } 399,5 \mathrm{mg} / \mathrm{L}^{*} \\
\text { 2. DQO: } 1476 \mathrm{mg} / \mathrm{L}^{*} \\
\text { 3. Nivel de } \mathrm{pH}: 6-11^{* *}\end{array}$ \\
Situación mejorada de & $\begin{array}{l}\text { 1. DBO: }<399,5 \mathrm{mg} / \mathrm{L} \\
\text { los ecoindicadores }\end{array}$ \\
& $\begin{array}{l}\text { 2. DQO: }<1000 \mathrm{mg} / \mathrm{L} \\
\text { 3. Nivel de } \mathrm{pH}: 6-9\end{array}$ \\
\hline
\end{tabular}

* Valores extraídos del estudio de Monllor et al. (2013)

**Valores extraídos del estudio de Gómez Gallo (2008)

Elaboración propia

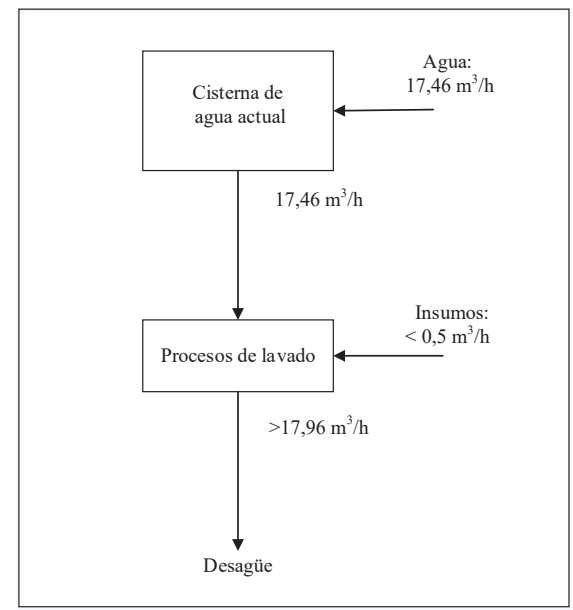

Figura 2. Sistema actual de los efluentes

Elaboración propia 


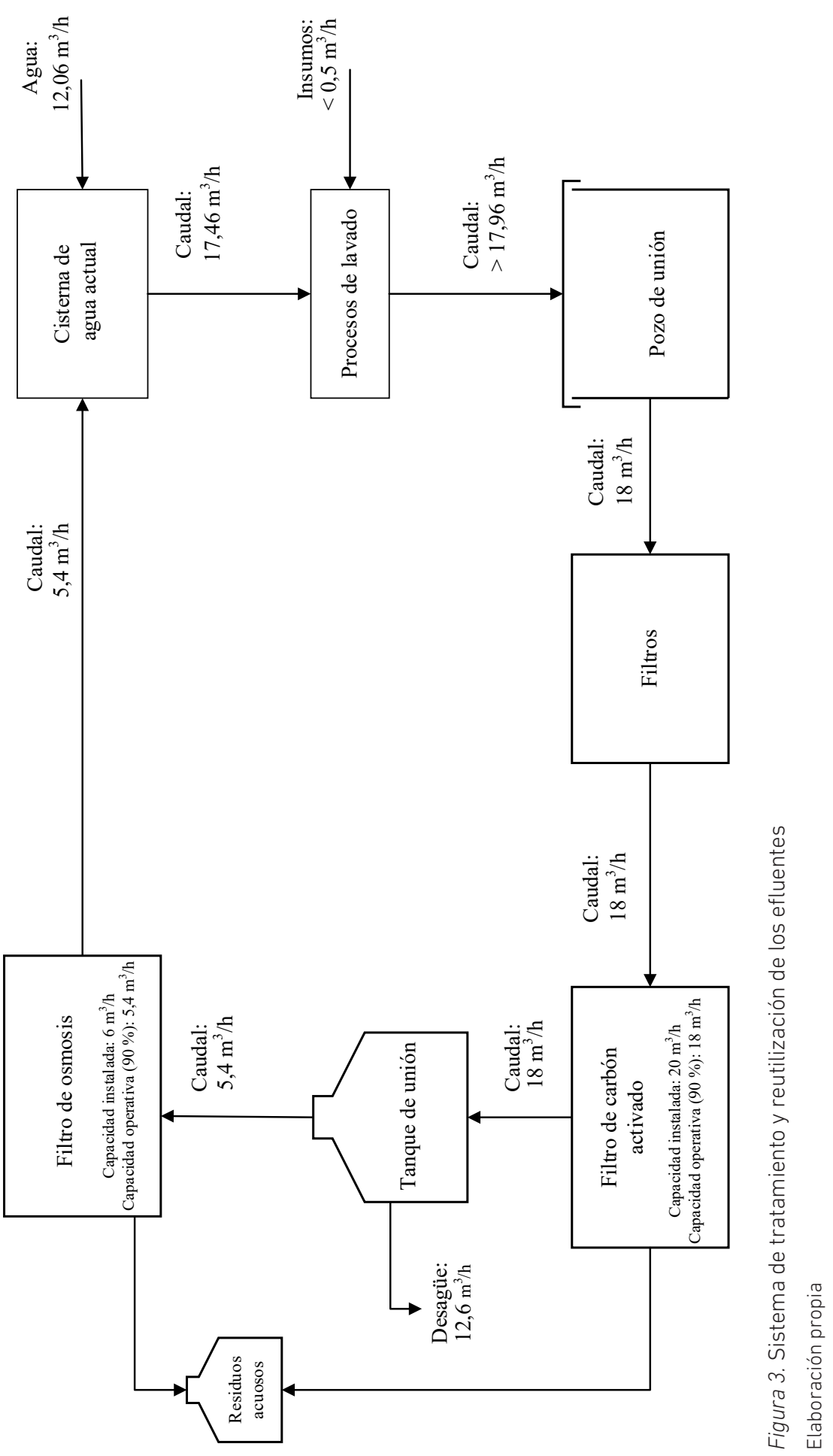




\section{CONCLUSIONES}

A continuación, se listan las conclusiones de las mejoras propuestas y de la metodología empleada.

- Gracias al empleo de la matriz IRA, se identificó que los aspectos e impactos ambientales generados por la empresa son diversos y significativos; ya que estos tienen diferentes niveles de impacto, pudiendo llegar a dañar, fuertemente, al medioambiente por emisiones de compuestos tóxicos y el empleo de químicos tóxicos.

- Se ha demostrado con la implementación de nuevas tecnologías, como el uso de máquinas láser, que puede eliminarse totalmente el impacto generado en el proceso de focalizado, ya que el ecoindicador del proceso tendría un valor de cero (0).

- En el proceso de secado, mejorando y manteniendo la infraestructura en buen estado, se mitiga el impacto en su totalidad.

- Gracias al sistema de tratamiento de efluentes, se puede mitigar el impacto generado en el proceso de lavado y centrifugado, cumplimiento con los indicadores de VMA para efluentes no domésticos.

- El tema de las capacitaciones y la concientización del personal es de mucha importancia, puesto que se mejora el indicador de reprocesos en la operación de lavado.

- Al implementar todas las mejoras propuestas, a pesar de conllevar una inversión, representaría menor impacto ambiental, mejorando así, de manera integral, el modelo de negocio actual de la empresa, llegando a ser un modelo de negocio sostenible.

\section{REFERENCIAS}

Brandon C. A., Johnson, J. S., Minturn, R. E., y Porter, J. J. (1973). Complete reuse of textile dyeing wastes processed with dynamic membrane hyperfiltration. Textile Chemist \& Colorist, 5(7), 35.

Chauvet, S. B., Bello B. E., Barnes N., y Albarracín P. M. (2014). Evaluación de aspectos ambientales: una adaptación de un método de riesgos de accidentes. Revista Argentina de Ingeniería, 3, 69-75. Recuperado de https://radi.org.ar/wp-content/ uploads/2016/08/13-EVALUACION-ASPECTOS-AMBIENTALES.Secci+\%C2\%A6nIngenier+\%C2\%A1a-Sostenible.pdf

Cueva, F. D. (2014). Efectos del cambio climático en la economía, el comercio internacional y la estrategia empresarial. Contabilidad y Negocios, 9(18), 75-98. Recuperado de http://revistas.pucp.edu.pe/index.php/contabilidadynegocios/article/view/11613 
Decreto Supremo n. ${ }^{\circ}$ 021-2009-VIVIENDA. Aprueban valores máximos admisibles (VMA) de las descargas de aguas residuales no doméstica en el sistema de alcantarillado sanitario. (20 de noviembre del 2009). Recuperado de http:// www3.vivienda.gob.pe/direcciones/Documentos/DS_2009_021.pdf

DeJohn, P., y Hutchins, R. (1976). Treatment of dye wastes with granular activated carbon. Textile Chemist \& Colorist, 8(4).

Dirección de Normalización (2015). Norma Técnica Peruana NTP-ISO 14001:2015. Instituto Nacional de Calidad.

Gómez Gallo, M. E. (2008). Reutilización de las aguas de la planta de tintorería de hilazas en la industria Calcetines Crystal S. A. Revista Producción + Limpia, 3(2), 50-57.

International Organization for Standardization. (2015). Norma Internacional ISO 14001.

Ley n. ${ }^{\circ}$ 28611. Ley General del Ambiente. (2005). Ministerio del Ambiente.

Monllor, P., Sanz, J. F., Vicente, R., y Bonet, M. (2013). Reuse in exhaust dyeing processes of textile wastewaters. Textile Technology Complete, 14(1), 163-166.

Montoya, W. M. (2019). Propuesta de un sistema de gestión ambiental para una lavandería industrial bajo la norma IS014001:2015 (tesis para optar el título profesional de ingeniero industrial). Pontificia Universidad Católica del Perú. Recuperado de http://tesis.pucp.edu.pe/repositorio/handle/20.500.12404/15571 
